Turkish Journal of Mathematics

http: / /journals.tubitak.gov.tr/math/
Turk J Math

(2019) 43: $224-240$

(C) TÜBİTAK

doi:10.3906/mat-1803-121

\title{
Sectional curvatures on Weyl manifolds with a special metric connection
}

Fatma ÖZDEMİ* (D), Mustafa Deniz TÜRKOĞLU(D)

Department of Mathematics, Faculty of Science and Letters, İstanbul Technical University, Maslak, İstanbul, Turkey
Received: 27.03 .2018

Accepted/Published Online: 29.11.2018

Final Version: 18.01.2019

\begin{abstract}
In this paper, Weyl manifolds, denoted by $W S(g, w, \pi, \mu)$, having a special a semisymmetric recurrentmetric connection are introduced and the uniqueness of this connection is proved. We give an example of $W S(g, w, \pi, \mu)$ with a constant scalar curvature. Furthermore, we define sectional curvatures of $W S(g, w, \pi, \mu)$ and prove that any isotropic Weyl manifold $W S(g, w, \pi, \mu)$ is locally conformal to an Einstein manifold with a semisymmetric recurrentmetric connection, $E W S(g, w, \pi, \mu)$.
\end{abstract}

Key words: Weyl manifold, semisymmetric connection, recurrent-metric connection, generalized Bianchi identities, sectional curvature

\section{Introduction}

Linear connections are defined on manifolds to establish the parallel transport of vector fields along any curve in the manifolds, so that infinitesimally close tangent spaces are connected to each other. Riemannian manifolds are defined by a linear metric connection or by the Levi-Civita connection, and this connection is uniquely defined once the metric tensor is determined or given and the metric tensor keeps the geometrical information about the space.

The concept of the semisymmetric linear connection in a differentiable manifold without metricity condition was introduced by Friedmann and Schouten in 1924 (see [4, p. 214]). Later, Hayden introduced the idea of metric connection with torsion on a Riemannian manifold in 1932 [5]. Afterwards, Yano considered the semisymmetric metric connection on a Riemannian manifold in 1970 [18].

Spaces with metric, nonmetric, torsion-free, or torsionful connections have wide applications in theories of gravity as well as differential geometry [16].

This paper is devoted to the study of Weyl manifolds endowed with a semisymmetric recurrent-metric connection, which we denote by $W S(g, w, \pi, \mu)$. We derive some relations involving the curvature tensor of a semisymmetric recurrent-metric connection. Moreover, we give an example of $W S(g, w, \pi, \mu)$ spaces with constant scalar curvature.

We also define the sectional curvature of Weyl manifolds with a semisymmetric recurrent-metric connection $W S(g, w, \pi, \mu)$ and we prove that any isotropic Weyl manifold with a semisymmetric recurrent-metricconnection can be locally conformal to an Einstein manifold with semisymmetric recurrent-metric connection $E W S(g, w, \pi, \mu)$.

*Correspondence: fozdemir@itu.edu.tr 2010 AMS Mathematics Subject Classification: Primary 53A30, 53A40 
ÖZDEMİR and TÜRKOĞLU/Turk J Math

\section{Preliminaries}

In this section, we first give some preliminary concepts related to Weyl spaces and semisymmetric spaces.

Two Riemannian metrics $g$ and $\bar{g}$ are conformal if they coincide up to a factor that is positive function, i.e. $\bar{g}=e^{2 \lambda} g$. This is an equivalence relation, each class $G$ being called a conformal structure. A Weyl structure is a map $w: G \rightarrow \Lambda^{1}(W)$ satisfying $w\left(e^{2 \lambda} g\right)=w(g)+2 d \lambda$. A manifold with a Weyl structure is called a Weyl manifold, denoted by $W(g, w)$.

In [3], it was proved that for a Weyl manifold $W(g, w)$, there exists a unique torsion-free connection $\nabla$ that preserves the conformal class $G$. Preserving the conformal class means that for any $g \in G$ there exists 1-form $w$ such that

$$
\nabla g=2 w \otimes g
$$

Equation (2.1) can be expressed in local coordinates as

$$
\nabla_{k} g_{i j}=2 w_{k} g_{i j}
$$

Here, $w$ is a 1 -form called a complementary covector field.

Under the renormalization of the metric tensor $g$,

$$
\bar{g}=\Omega^{2} g,(\Omega>0),
$$

the 1 -form $w$ is transformed by the law

$$
\bar{w}=w+d \ln \Omega,
$$

so that

$$
\nabla_{k} \bar{g}_{i j}=2 \bar{w}_{k} \bar{g}_{i j}
$$

Here, $\Omega$ is a positive scalar differentiable function defined on $W(g, w)$ (see [6] and [10, p. 152]).

The relation between the Weyl connection $\nabla$ and the Riemannian connection $\nabla^{g}$ is

$$
\nabla_{X} Y=\nabla_{X}^{g} Y-w(X) Y-w(Y) X+g(X, Y) \psi
$$

where $X, Y$ are vector fields on $W(g, w)$ and $\psi$ is the dual vector field to $w$ such that $w(X)=g(X, \psi)$.

In local coordinates, (2.6) can be given by

$$
\Gamma_{j i}^{h}=\left\{\begin{array}{l}
h \\
j i
\end{array}\right\}-\left(w_{j} \delta_{i}^{h}+w_{i} \delta_{j}^{h}-w^{h} g_{j i}\right),
$$

where $\Gamma_{j i}^{h}$ are the coefficients of the Weyl connection and

$$
\left\{\begin{array}{l}
h \\
j i
\end{array}\right\}=\frac{1}{2} g^{h m}\left(\partial_{j} g_{m i}+\partial_{i} g_{m j}-\partial_{m} g_{j i}\right)
$$

are the coefficients of the Levi-Civita connection $\nabla^{g}$ see $[2$, p. 81], and [10, p. 154].

The curvature tensor $W$ of $\nabla$ is given by

$$
W(X, Y) Z=\nabla_{X} \nabla_{Y} Z-\nabla_{Y} \nabla_{X} Z-\nabla_{[X, Y]} Z
$$


Using (2.6) in (2.9), the curvature tensor of $W(g, w)$ is obtained:

$$
W(X, Y) Z=R(X, Y) Z-s(X, Z) Y+s(Y, Z) X+s(Y, X) Z-s(Y, X) Z+g(Y, Z) \bar{S} X-g(X, Z) \bar{S} Y,
$$

for any vector fields $X, Y, Z$ where $R$ denotes the curvature tensor of the Riemannian connection $\nabla^{g}$ and $s$ is the tensor field of type $(0,2)$ defined by

$$
s(X, Y)=\left(\nabla_{X} w\right)(Y)+w(X) w(Y)-\frac{1}{2} w(\psi) g(X, Y),
$$

and $\bar{S}$ is the tensor field of type $(1,1)$ defined by

$$
g(\bar{S} X, Y)=s(X, Y)
$$

In local coordinates, using the curvature tensor of $W(g, w)$,

$$
W_{k j i}^{h}=\partial_{k} \Gamma_{j i}^{h}-\partial_{j} \Gamma_{k i}^{h}-\Gamma_{k i}^{t} \Gamma_{j t}^{h}+\Gamma_{j i}^{t} \Gamma_{k t}^{h}
$$

we obtain the components of curvature tensor $W_{k j i}{ }^{h}$ as

$$
W_{k j i}^{h}=R_{k j i}^{h}-w_{k i} \delta_{j}^{h}+w_{j i} \delta_{k}^{h}+\left(w_{j k}-w_{k j}\right) \delta_{i}^{h}+g^{h s}\left(w_{k s} g_{j i}-w_{j s} g_{k i}\right)
$$

with

$$
w_{j i}=\nabla_{j} w_{i}+w_{j} w_{i}-\frac{1}{2} w_{t} w^{t} g_{j i}
$$

and $R_{k j i}{ }^{h}$ represents the Riemannian curvature tensor with respect to the Levi-Civita connection.

The curvature tensor and covariant curvature tensor, the Ricci tensor, and the scalar curvature of Weyl space are defined through parallel transportation of vector fields $v$, respectively, by

$$
\begin{aligned}
& W_{k j i l}=W_{k j i}{ }^{m} g_{m l}, \\
& W_{j i}=g^{k l} W_{k j i l}=W_{l j i}{ }^{l}, \\
& W=g^{i j} W_{i j} .
\end{aligned}
$$

By transvecting (2.14) with $g_{h m}$, we get

$$
W_{k j i m}=R_{k j i m}-w_{k i} g_{j m}+w_{j i} g_{k m}+\left(w_{j k}-w_{k j}\right) g_{i m}+w_{k m} g_{j i}-w_{j m} g_{k i},
$$

which is called the covariant curvature tensor of $W(g, w)$.

The curvature tensor, the covariant curvature tensor, and the Ricci tensor of $W(g, w)$ satisfy the following symmetry properties (see [10, p. 157]):

$$
\begin{aligned}
& W_{k j i l}=-W_{j k i l}, \\
& W_{k j i l}+W_{k j l i}=2 g_{i l}\left(\nabla_{j} w_{k}-\nabla_{k} w_{j}\right)=4 g_{i l} \nabla_{[j} w_{k]} .
\end{aligned}
$$


By using symmetries of the curvature tensor of Weyl space, we obtain identities that are similar to identities held in Riemannian spaces. The following identities are known as the first and second Bianchi identities for Weyl spaces, respectively [6, 11]:

$$
\begin{aligned}
& W_{k j i}{ }^{l}+W_{j i k}{ }^{l}+W_{i k j}{ }^{l}=0, \\
& \nabla_{m} W_{k j i}{ }^{l}+\nabla_{k} W_{j m i}{ }^{l}+\nabla_{j} W_{m k i}{ }^{l}=0 .
\end{aligned}
$$

Furthermore, the Ricci tensor of the Weyl manifold is computed in terms of Ricci curvature $R_{k j}$ of Riemannian space as

$$
W_{k j}=R_{k j}+(n-2) w_{k j}+\left(w_{k j}-w_{j k}\right)+w_{s t} g^{s t} g_{k j},
$$

where $w_{k j}$ is defined in (2.15). It should also be noted that the Ricci tensor of a Weyl manifold is not symmetric; its symmetric and antisymmetric parts are given as follows (see [2], p.82):

$$
\begin{gathered}
W_{(k j)}=R_{k j}+\frac{1}{2}(n-2)\left[\nabla_{j} w_{k}+\nabla_{k} w_{j}+2 w_{k} w_{j}-2 w_{t} w^{t} g_{j k}\right]+g_{j k} \nabla_{t} w^{t}, \\
W_{[k j]}=n \nabla_{[k} w_{j]} .
\end{gathered}
$$

From (2.24), we obtain the scalar curvature of Weyl space:

$$
W=R+2(n-1) \nabla_{j} w^{j}-(n-1)(n-2) w_{j} w^{j}
$$

where $R$ is the Riemannian scalar curvature and $w$ is the complementary covector defined in (2.2).

In the next section, we give some definitions and properties of manifolds with semisymmetric connection, and we also construct a new special connection on a Weyl manifold.

\section{Semisymmetric recurrent-metric connection on Weyl manifolds}

In the literature, the idea of semisymmetric connection was introduced by $[1,13,15,17,18]$ and curvaturerelated properties were studied widely therein. Let $M$ be an $n$-dimensional, $(n>2)$ differentiable manifold. A linear connection $\nabla^{*}$ on $M$, whose coefficients are $\Gamma^{*}{ }_{j k}^{i}$, is said to be semisymmetric if the torsion tensor $T$ of $\nabla^{*}$ satisfies the relation

$$
T(X, Y)=\pi(Y) X-\pi(X) Y,
$$

where $\pi$ is a 1-form, and $X, Y$ are smooth vector fields on $M$. In local coordinates, (3.1) can be written as

$$
T_{j k}^{i}=\Gamma_{j k}^{*}-\Gamma_{k j}^{*}{ }_{k j}^{i}=\pi_{k} \delta_{j}^{i}-\pi_{j} \delta_{k}^{i} .
$$

In addition, if a semisymmetric connection has the recurrency condition

$$
\nabla_{X}^{*} g=2 \mu(X) g
$$


in local coordinates, (3.3) can be written as

$$
\nabla_{k}^{*} g_{i j}=2 \mu_{k} g_{i j}
$$

and then the connection $\nabla^{*}$ is said to be a semisymmetric recurrent-metric connection and $\mu$ is called the recurrent covariant vector field $[7,8]$.

In this work, we use the notion of a semisymmetric recurrent metric connection for Weyl manifolds. Let $\bar{\nabla}$ be a linear connection with coefficients $\bar{\Gamma}_{j k}^{i}$ on a Weyl manifold $W(g, w)$ satisfying (3.2). If the following relation also holds on $W(g, w)$,

$$
\bar{\nabla}_{X} g(Y, Z)=2(w+\mu)(X) g(Y, Z)
$$

in local coordinates, (3.5) is represented by

$$
\bar{\nabla}_{k} g_{i j}=\nabla_{k} g_{i j}+2 \mu_{k} g_{i j}=2\left(w_{k}+\mu_{k}\right) g_{i j}
$$

and then $W(g, w)$ is called a Weyl manifold with a semisymmetric recurrent-metric connection denoted by $W S(g, w, \pi, \mu)$.

From (2.2), we have

$$
\begin{aligned}
\nabla_{k} g_{i j} & =\partial_{k} g_{i j}-g_{h j} \Gamma_{k i}^{h}-g_{i h} \Gamma_{k j}^{h} \\
& =2 w_{k} g_{i j}
\end{aligned}
$$

and from (3.6), more explicitly,

$$
\begin{aligned}
\bar{\nabla}_{k} g_{i j} & =\partial_{k} g_{i j}-g_{h j} \bar{\Gamma}_{k i}{ }^{h}-g_{i h} \bar{\Gamma}_{k j}{ }^{h} \\
& =2\left(w_{k}+\mu_{k}\right) g_{i j} .
\end{aligned}
$$

By using (3.8), we have

$$
\bar{\nabla}_{k} g^{i j}=-2\left(w_{k}+\mu_{k}\right) g^{i j}
$$

Here, we will examine the existence and uniqueness of the semisymmetric recurrent-metric connection $\bar{\nabla}$ on a Weyl manifold and will prove the following theorem.

Theorem 3.1 Let $W S(g, w, \pi, \mu)$ be an n-dimensional Weyl manifold equipped with the semisymmetric recurrentmetric connection $\bar{\nabla}$ associated with 1 -forms $w, \pi$, and $\mu$ satisfying (2.2), (3.2), and (3.4), respectively. Then there exists a unique connection $\bar{\nabla}$ on $W S(g, w, \pi, \mu)$ given by

$$
\bar{\nabla}_{X} Y=\nabla_{X} Y-\mu(X) Y-\mu(Y) X+g(X, Y) \xi+\pi(Y) X-g(X, Y) \eta,
$$

where $\xi$ and $\eta$ are dual vector fields such that

$$
\mu(X)=g(X, \xi), \quad \pi(X)=g(X, \eta) .
$$


Proof Let $\bar{\nabla}$ be a semisymmetric recurrent metric connection and $\nabla$ be a Weyl connection. We have

$$
\left(\bar{\nabla}_{X} g\right)(Y, Z)=\bar{\nabla}_{X} g(Y, Z)-g\left(\bar{\nabla}_{X} Y, Z\right)-g\left(Y, \bar{\nabla}_{X} Z\right)
$$

and

$$
\left(\nabla_{X} g\right)(Y, Z)=\nabla_{X} g(Y, Z)-g\left(\nabla_{X} Y, Z\right)-g\left(Y, \nabla_{X} Z\right)
$$

for any vector fields $X, Y$, and $Z$.

We put

$$
\bar{\nabla}_{X} Y=\nabla_{X} Y+U(X, Y),
$$

where $U$ is a tensor field of type $(1,2)$ defined as the difference of the connections.

Using (3.1) and (3.14) it is obtained that

$$
\begin{aligned}
T(X, Y) & =\bar{\nabla}_{X} Y-\bar{\nabla}_{Y} X-[X, Y], \\
& =U(X, Y)-U(Y, X) .
\end{aligned}
$$

From (3.12), (3.13), (3.14), and (3.15) we get

$$
g(U(X, Y), Z)+g(U(X, Z), Y)=-2 \mu(X) g(Y, Z) .
$$

By using (3.15), and permuting vector fields $X, Y$, and $Z$ for $T$, we get

$$
\begin{gathered}
g(T(X, Y), Z)=g(U(X, Y), Z)-g(U(Y, X), Z), \\
g(T(Z, X), Y)=g(U(Z, X), Y)-g(U(X, Z), Y), \\
g(T(Z, Y), X)=g(U(Z, Y), X)-g(U(Y, Z), X) .
\end{gathered}
$$

From (3.16) and (3.17) we obtain

$$
\begin{aligned}
g(T(X, Y), Z)+g(T(Z, X), Y)+g(T(Z, Y), X) & =2 g(U(X, Y), Z)+2 \mu(X) g(Y, Z) \\
& +2 \mu(Y) g(Z, X)-2 \mu(Z) g(X, Y) .
\end{aligned}
$$

Defining the tensor $\dot{T}$ of type $(1,2)$ as

$$
g(T(Z, X), Y)=g(\dot{T}(X, Y), Z)
$$

equation (3.18) can be written as

$$
\begin{aligned}
g(U(X, Y), Z)= & \frac{1}{2}[g(T(X, Y), Z)+g(\dot{T}(X, Y), Z)+g(\dot{T}(Y, X), Z)] \\
& -\mu(X) g(Y, Z)-\mu(Y) g(Z, X)+\mu(Z) g(X, Y)
\end{aligned}
$$

Thus, we find

$$
U(X, Y)=\frac{1}{2}[T(X, Y)+\dot{T}(X, Y)+\dot{T}(Y, X)]-\mu(X) Y-\mu(Y) X+g(X, Y) \xi,
$$

where $\mu(X)=g(X, \xi)$. 
From (3.1) and (3.19) we have

$$
\begin{aligned}
g(T(Z, X), Y) & =g(\pi(X) Z, Y)-g(\pi(Z) X, Y), \\
& =g(\dot{T}(X, Y), Z) .
\end{aligned}
$$

From (3.19), and (3.22), we reach

$$
g(\dot{T}(X, Y), Z)=\pi(X) g(Z, Y)-g(Z, \eta) g(X, Y),
$$

which implies

$$
\dot{T}(X, Y)=\pi(X) Y-g(X, Y) \eta,
$$

where $\pi(X)=g(X, \eta)$.

Hence, (3.21) turns into

$$
U(X, Y)=\pi(Y) X-\mu(X) Y-\mu(Y) X+g(X, Y) \xi-g(X, Y) \eta .
$$

Then (3.14) becomes

$$
\bar{\nabla}_{X} Y=\nabla_{X} Y-\mu(X) Y-\mu(Y) X+g(X, Y) \xi+\pi(Y) X-g(X, Y) \eta,
$$

which completes the proof.

Also, equation (3.10) is obtained in local coordinates as

$$
\bar{\Gamma}_{i k}^{l}=\left\{\begin{array}{c}
l \\
i k
\end{array}\right\}-\left(w_{i} \delta_{k}^{l}+w_{k} \delta_{i}^{l}-w^{l} g_{i k}\right)+\left(\lambda_{k} \delta_{i}^{l}-\mu_{i} \delta_{k}^{l}-\lambda^{l} g_{i k}\right),
$$

where $\lambda_{k}=\pi_{k}-\mu_{k}$.

The following subsection is devoted to presentation of curvature tensors of Weyl manifolds with the semisymmetric recurrent-metric connection, $W S(g, w, \pi, \mu)$, in local coordinates in detail. The covariant curvature tensor, the Ricci tensor, and the scalar curvature of $W S(g, w, \pi, \mu)$ will be denoted by $\bar{R}_{k j i m}, \bar{R}_{j i}$, and $\bar{R}$, respectively.

\subsection{The curvature tensor of $W S(g, w, \pi, \mu)$}

Theorem 3.2 The curvature tensor of a Weyl manifold with the semisymmetric recurrent-metric connection $W S(g, w, \pi, \mu)$ has the following properties:

$$
\text { (i) } \bar{R}_{k j i m}=W_{k j i m}+Q_{k j i m}-\alpha_{i j} g_{m k}+\alpha_{i k} g_{m j}-\alpha_{m k} g_{i j}+\alpha_{m j} g_{i k},
$$

where $W_{\text {kjim }}$ is the covariant curvature tensor of $W(g, w)$, and

$$
Q_{k j i}^{h}=\lambda_{k i} \delta_{j}^{h}-\lambda_{j i} \delta_{k}^{h}+\lambda_{j l} g^{l h} g_{k i}-\lambda_{k l} g^{l h} g_{j i}+\delta_{i}^{h}\left(\nabla_{j} \mu_{k}-\nabla_{k} \mu_{j}\right),
$$

or by transvecting with metric tensor $g_{h m}$

$$
Q_{k j i m}=g_{m j} \lambda_{k i}-g_{m k} \lambda_{j i}+\lambda_{j m} g_{k i}-\lambda_{k m} g_{j i}+2 g_{m i} \nabla_{[j} \mu_{k]},
$$


and

$$
\begin{array}{r}
\alpha_{i j}=\lambda_{i} w_{j}+\lambda_{j} w_{i}-w_{l} \lambda^{l} g_{i j}, \\
\lambda_{k i}=\nabla_{k} \lambda_{i}-\lambda_{k} \lambda_{i}+\frac{1}{2} g_{k i} \lambda_{t} \lambda^{t},
\end{array}
$$

and also, $\lambda, w$, and $\mu$ are 1 -forms as given in (3.2), (2.2), and (3.4), respectively.

$$
\text { (ii) } \bar{R}_{j i}=W_{j i}+Q_{j i}-(n-2)\left(\lambda_{j} w_{i}+\lambda_{i} w_{j}-2 g_{j i} w_{t} \lambda^{t}\right)
$$

where $W_{j i}$ is given by (2.24), and

$$
\begin{aligned}
& Q_{j i}=Q_{k j i m} g^{k m}=(n-2)\left[-\nabla_{j} \lambda_{i}+\lambda_{i} \lambda_{j}-g_{j i} \lambda_{t} \lambda^{t}\right]-g_{j i} \nabla_{t} \lambda^{t}+2 \nabla_{[j} \mu_{i]} . \\
& \text { (iii) } \bar{R}=R+2(n-1)\left(\nabla_{t} w^{t}-\nabla_{t} \lambda^{t}\right)-(n-1)(n-2)\left(w_{t}-\lambda_{t}\right)\left(w^{t}-\lambda^{t}\right),
\end{aligned}
$$

where $R$ is the Riemannian scalar curvature and holds the relation $R=R_{j i} g^{j i}$.

\section{Proof}

(i) In spaces with torsion, parallel transport of vector fields is defined by [18]. The curvature tensor of $W S(g, w, \pi, \mu)$ can be computed by using the Ricci identity for a covariant vector field $v_{i}$ :

$$
\left(\bar{\nabla}_{k} \bar{\nabla}_{j}-\bar{\nabla}_{j} \bar{\nabla}_{k}\right) v_{i}=-\bar{R}_{k j i}{ }^{t} v_{t}-T_{k j}{ }^{t} \bar{\nabla}_{t} v_{i}
$$

where $\bar{R}_{k j i}{ }^{h}$ is the curvature tensor of $W S(g, w, \pi, \mu)$,

$$
\bar{R}_{k j i}{ }^{h}=\partial_{k} \bar{\Gamma}_{j i}{ }^{h}-\partial_{j} \bar{\Gamma}_{k i}{ }^{h}+\bar{\Gamma}_{j i}{ }^{t} \bar{\Gamma}_{k t}{ }^{h}-\bar{\Gamma}_{k i}{ }^{t} \bar{\Gamma}_{j t}{ }^{h}
$$

and $T_{i j}{ }^{h}$ is the torsion tensor of $W S(g, w, \pi, \mu)$,

$$
T_{i j}{ }^{h}=\bar{\Gamma}_{i j}{ }^{h}-\bar{\Gamma}_{j i}{ }^{h}=U_{i j}{ }^{h}-U_{j i}{ }^{h} .
$$

Substituting coefficients of connections (3.26) in (3.36), and after some calculations, we obtain the curvature tensor of $W S(g, w, \pi, \mu)$ as

$$
\begin{array}{r}
\bar{R}_{k j i}{ }^{h}=W_{k j i}{ }^{h}+Q_{k j i}{ }^{h}-\delta_{k}^{h}\left(\lambda_{j} w_{i}+\lambda_{i} w_{j}-w_{l} \lambda^{l} g_{i j}\right)+\delta_{j}^{h}\left(\lambda_{k} w_{i}+\lambda_{i} w_{k}-w_{l} \lambda^{l} g_{i k}\right) \\
-g_{i j}\left(\lambda_{k} w^{h}+\lambda^{h} w_{k}-w_{l} \lambda^{l} \delta_{k}^{h}\right)+g_{i k}\left(\lambda_{j} w^{h}+\lambda^{h} w_{j}-w_{l} \lambda^{l} \delta_{j}^{h}\right),
\end{array}
$$

where $W_{k j i}{ }^{h}$ represents the curvature tensor of Weyl space defined in (2.14).

If we simplify our calculations we define the tensor $Q_{k j i}{ }^{h}$ as in (3.28) and $\lambda_{k i}$ as in (3.31), respectively:

$$
Q_{k j i}^{h}=\delta_{j}^{h} \lambda_{k i}-\delta_{k}^{h} \lambda_{j i}+\lambda_{j l} g^{l h} g_{k i}-\lambda_{k l} g^{l h} g_{j i}+\delta_{i}^{h}\left(\nabla_{j} \mu_{k}-\nabla_{k} \mu_{j}\right)
$$


ÖZDEMİR and TÜRKOĞLU/Turk J Math

$$
\lambda_{k i}=\nabla_{k} \lambda_{i}-\lambda_{k} \lambda_{i}+\frac{1}{2} g_{k i} \lambda_{t} \lambda^{t}
$$

and multiplying (3.28) by the metric tensor $g_{h m}$, we obtain

$$
Q_{k j i m}=g_{m j} \lambda_{k i}-g_{m k} \lambda_{j i}+\lambda_{j m} g_{k i}-\lambda_{k m} g_{j i}+2 g_{m i} \nabla_{[j} \mu_{k]},
$$

and similarly, we get

$$
Q_{j i}=Q_{k j i m} g^{k m}=(n-2)\left[-\nabla_{j} \lambda_{i}+\lambda_{i} \lambda_{j}-g_{j i} \lambda_{t} \lambda^{t}\right]-g_{j i} \nabla_{t} \lambda^{t}+2 \nabla_{[j} \mu_{i]} .
$$

From (3.29), we see that the following antisymmetry property holds for $Q_{k j i l}$ :

$$
Q_{k j i l}=-Q_{j k i l}
$$

Multiplying (3.38) by metric tensor $g_{h m}$ and using (3.29) and (2.19), we reach (3.27):

$$
\bar{R}_{k j i m}=W_{k j i m}+Q_{k j i m}-g_{m k} \alpha_{i j}+g_{m j} \alpha_{i k}-g_{i j} \alpha_{m k}+g_{i k} \alpha_{m j}
$$

where

$$
\alpha_{i j}=\lambda_{i} w_{j}+\lambda_{j} w_{i}-g_{i j} w_{l} \lambda^{l}
$$

(ii) Now let us examine the Ricci curvature and its symmetric properties for $W S(g, w, \pi, \mu)$. Multiplying (3.27) by $g^{m k}$, we get the Ricci tensor of $W S(g, w, \pi, \mu)$ as

$$
\bar{R}_{j i}=W_{j i}+Q_{j i}-(n-2)\left(\lambda_{j} w_{i}+\lambda_{i} w_{j}\right)+2(n-2) g_{j i} w_{l} \lambda^{l},
$$

where $W_{j i}$ and $Q_{j i}$ are given by (2.24) and (3.33), respectively.

It is seen that the Ricci tensor $\bar{R}_{j i}$ of $W S(g, w, \pi, \mu)$ is not symmetric. The symmetric and antisymmetric parts of $\bar{R}_{j i}$ can be calculated as

$$
\begin{aligned}
\bar{R}_{(j i)} & =W_{(j i)}-\frac{(n-2)}{2}\left[\left(\nabla_{j} \lambda_{i}+\nabla_{i} \lambda_{j}\right)-2 \lambda_{i} \lambda_{j}+2 g_{j i} \lambda_{t} \lambda^{t}+2\left(\lambda_{j} w_{i}+\lambda_{i} w_{j}\right)\right] \\
& +g_{j i}\left[2(n-2) w_{t} \lambda^{t}-\nabla_{t} \lambda^{t}\right]
\end{aligned}
$$

and

$$
\bar{R}_{[j i]}=n \nabla_{[j} w_{i]}-(n-2) \nabla_{[j} \lambda_{i]}+2 \nabla_{[j} \mu_{i]} .
$$

(iii) Transvecting the Ricci curvature tensor $\bar{R}_{j i}$ in (3.40) by the metric tensor $g^{j i}$, we obtain

$$
\bar{R}=\bar{R}_{j i} g^{j i}
$$

and then the scalar curvature of $W S(g, w, \pi, \mu)$ is

$$
\bar{R}=R+2(n-1)\left(\nabla_{t} w^{t}-\nabla_{t} \lambda^{t}\right)-(n-1)(n-2)\left(w_{t}-\lambda_{t}\right)\left(w^{t}-\lambda^{t}\right) .
$$


ÖZDEMİR and TÜRKOĞLU/Turk J Math

Now we examine the properties of the covariant curvature tensor of Weyl space with the semisymmetric recurrent-metric connection $W S(g, w, \pi, \mu)$. Using the properties of $R_{k j i l}$ and $W_{k j i l}$, it can be seen that the curvature tensor of $W S(g, w, \pi, \mu)$ satisfies the following symmetry relations in the following propositions:

Proposition 3.3 The curvature tensor of $W S(g, w, \pi, \mu)$ satisfies the following symmetry relations:

$$
\begin{aligned}
\text { (i). } & \bar{R}_{k j i m} & =-\bar{R}_{j k i m}, \\
\text { (ii). } & \bar{R}_{k j i m}+\bar{R}_{k j m i} & =4 g_{i m}\left(\nabla_{[j} \mu_{k]}+\nabla_{[j} w_{k]}\right) .
\end{aligned}
$$

\section{Proof}

(i) Interchanging the indices $k$ and $j$ in equation (3.27), we have

$$
\bar{R}_{k j i m}+\bar{R}_{j k i m}=W_{k j i m}+W_{j k i m}+Q_{k j i m}+Q_{j k i m},
$$

and using (2.20) and (3.39) in the above equation, we get (3.45).

(ii) Using (2.20), (2.21), (3.29), and (3.30) in the equation of (3.27), we obtain (3.46).

$$
\begin{aligned}
\bar{R}_{k j i m}+\bar{R}_{k j m i} & =W_{k j i m}+W_{k j m i}+Q_{k j i m}+Q_{k j m i} \\
& =W_{k j i m}+W_{k j m i}+2 g_{i m}\left(\mu_{j k}-\mu_{k j}\right) \\
& =2 g_{i m}\left(\nabla_{[j} w_{k]}-\nabla_{[k} w_{j]}\right)+2 g_{i m}\left(\mu_{j k}-\mu_{k j}\right) \\
& =4 g_{i m}\left(\nabla_{[j} \mu_{k]}+\nabla_{[j} w_{k]}\right) .
\end{aligned}
$$

Note that if $w_{k}$ and $\mu_{k}$ are gradients or if $w_{k}$ and $\mu_{k}$ have opposite signs, then

$$
\bar{R}_{k j i m}=-\bar{R}_{k j m i} .
$$

In the following proposition, we introduce extended (generalized) first and second Bianchi identities for $W S(g, w, \pi, \mu)$.

Proposition 3.4 The curvature tensor of $W S(g, w, \pi, \mu)$ satisfies the following first and second Bianchi identities for $W S(g, w, \pi, \mu)$, respectively:

$$
\begin{gathered}
\text { (i). } \bar{R}_{k j i}{ }^{l}+\bar{R}_{j i k}{ }^{l}+\bar{R}_{i k j}{ }^{l}=2\left(\delta_{j}^{l} \nabla_{[k} \pi_{i]}+\delta_{i}^{l} \nabla_{[j} \pi_{k]}+\delta_{k}^{l} \nabla_{[i} \pi_{j]}\right), \\
\text { (ii). } \quad\left(\bar{\nabla}_{l} \bar{R}_{k j i}{ }^{t}+\bar{\nabla}_{k} \bar{R}_{j l i}{ }^{t}+\bar{\nabla}_{j} \bar{R}_{l k i}{ }^{t}\right)=2\left(\pi_{l} \bar{R}_{k j i}{ }^{t}+\pi_{k} \bar{R}_{j l i}{ }^{t}+\pi_{j} \bar{R}_{l k i}{ }^{t}\right) .
\end{gathered}
$$

\section{Proof}

(i) Using (3.38) and (3.31), and by changing indices $k, j, i$ cyclically, we get

$$
\bar{R}_{k j i}{ }^{l}+\bar{R}_{j i k}{ }^{l}+\bar{R}_{i k j}{ }^{l}=Q_{k j i}{ }^{l}+Q_{j i k}{ }^{l}+Q_{i k j}{ }^{l} .
$$


On the other hand, using (3.31), we calculate ${Q_{k j i}}^{l}+Q_{j i k}{ }^{l}+Q_{i k j}{ }^{l}$ as

$$
\begin{aligned}
Q_{k j i}{ }^{l}+Q_{j i k}{ }^{l}+Q_{i k j}{ }^{l} & =\delta_{j}^{l}\left(\lambda_{k i}-\lambda_{i k}\right)+\delta_{k}^{l}\left(\lambda_{i j}-\lambda_{j i}\right)+\delta_{i}^{l}\left(\lambda_{j k}-\lambda_{k j}\right) \\
& +\delta_{i}^{l}\left(\mu_{j k}-\mu_{k j}\right)+\delta_{k}^{l}\left(\mu_{i j}-\mu_{j i}\right)+\delta_{j}^{l}\left(\mu_{k i}-\mu_{i k}\right),
\end{aligned}
$$

where

$$
\mu_{k j}=\nabla_{k} \mu_{j}-\mu_{j} \mu_{k}+\frac{1}{2} g_{j k} \mu_{t} \mu^{t}
$$

Using (3.31), and arranging (3.50), we find

$$
\begin{aligned}
Q_{k j i}{ }^{l}+Q_{j i k}{ }^{l}+Q_{i k j}{ }^{l} & =\delta_{j}^{l}\left[\nabla_{k}\left(\pi_{i}-\nabla_{i} \pi_{k}\right)\right] \\
& +\delta_{k}^{l}\left[\nabla_{i}\left(\pi_{j}-\nabla_{j} \pi_{i}\right)\right]+\delta_{i}^{l}\left[\nabla_{j}\left(\pi_{k}-\nabla_{k} \pi_{j}\right)\right]
\end{aligned}
$$

Using (3.52) in (3.49), we get

$$
\bar{R}_{k j i}{ }^{l}+\bar{R}_{j i k}{ }^{l}+\bar{R}_{i k j}{ }^{l}=2\left(\delta_{j}^{l} \nabla_{[k} \pi_{i]}+\delta_{i}^{l} \nabla_{[j} \pi_{k]}+\delta_{k}^{l} \nabla_{[i} \pi_{j]}\right)
$$

which is called the generalized first Bianchi identity.

Also, the covariant form is obtained:

$$
\bar{R}_{k j i m}+\bar{R}_{j i k m}+\bar{R}_{i k j m}=2\left(g_{j m} \nabla_{[k} \pi_{i]}+g_{i m} \nabla_{[j} \pi_{k]}+g_{k m} \nabla_{[i} \pi_{j]}\right) .
$$

(ii) Using the Ricci identity (3.35) and differentiating covariantly both sides of (3.35), we get

$$
\begin{aligned}
-\bar{\nabla}_{l} \bar{\nabla}_{k} \bar{\nabla}_{j} v_{i}+\bar{\nabla}_{l} \bar{\nabla}_{j} \bar{\nabla}_{k} v_{i} & =\bar{\nabla}_{l}\left(\bar{R}_{k j i}{ }^{t}\right) v_{t}+\bar{R}_{k j i}{ }^{t} \bar{\nabla}_{l}\left(v_{t}\right) \\
& +\bar{\nabla}_{l}\left(T_{k j}{ }^{t}\right) \bar{\nabla}_{t} v_{i}+T_{k j}{ }^{t}\left(\bar{\nabla}_{l} \bar{\nabla}_{t} v_{i}\right),
\end{aligned}
$$

which is written in terms of the covariant derivative of the curvature tensor and torsion tensor.

Now, interchanging the indices $l, k$, and $j$ in (3.53), using the components of the torsion tensor of (3.2), and by some tensor calculations, the Ricci identity (3.35) reduces to

$$
\begin{aligned}
& T_{l k}{ }^{t}\left(\bar{\nabla}_{t} \bar{\nabla}_{j}-\bar{\nabla}_{j} \bar{\nabla}_{t}\right) v_{i}+T_{j l}{ }^{t}\left(\bar{\nabla}_{t} \bar{\nabla}_{k}-\bar{\nabla}_{k} \bar{\nabla}_{t}\right) v_{i}+T_{k j}{ }^{t}\left(\bar{\nabla}_{t} \bar{\nabla}_{l}-\bar{\nabla}_{l} \bar{\nabla}_{t}\right) v_{i} \\
& \quad=-2 \pi_{k}\left(\bar{R}_{l j i}{ }^{t} v_{t}+T_{l j}{ }^{t} \bar{\nabla}_{t} v_{i}\right)-2 \pi_{l}\left(\bar{R}_{j k i}{ }^{t} v_{t}+T_{j k}{ }^{t} \bar{\nabla}_{t} v_{i}\right)-2 \pi_{j}\left(\bar{R}_{k l i}{ }^{t} v_{t}+T_{k l}{ }^{t} \bar{\nabla}_{t} v_{i}\right) .
\end{aligned}
$$

Using (3.53), (3.54), and (3.2), we find

$$
\left(\bar{\nabla}_{l} \bar{R}_{k j i}{ }^{t}+\bar{\nabla}_{k} \bar{R}_{j l i}{ }^{t}+\bar{\nabla}_{j} \bar{R}_{l k i}{ }^{t}\right)=2\left(\pi_{l} \bar{R}_{k j i}{ }^{t}+\pi_{k} \bar{R}_{j l i}{ }^{t}+\pi_{j} \bar{R}_{l k i}{ }^{t}\right),
$$

which is called the generalized second Bianchi identity for $W S(g, w, \pi, \mu)$. 
Theorem 3.5 $W S(g, w, \pi, \mu)$ and $W(g, w)$ have the same curvature tensors if and only if the recurrent covariant vector field $\mu_{k}$ of $\bar{\nabla}$ defined by (3.4) is a gradient vector and the following equation holds:

$$
\lambda_{i j}+\alpha_{j i}=0
$$

where $\alpha_{i j}$ and $\lambda_{i j}$ are as in (3.30) and (3.31), respectively.

Proof Let $W S(g, w, \pi, \mu)$ and $W(g, w)$ have the same curvature tensors:

$$
\bar{R}_{k j i m}=W_{k j i m}
$$

Using (3.27), we have

$$
Q_{k j i m}=g_{m k} \alpha_{i j}-g_{m j} \alpha_{i k}+g_{i j} \alpha_{m k}-g_{i k} \alpha_{m j}
$$

Also, from (3.30), (3.31), and (3.29), we obtain the relation

$$
\begin{aligned}
g_{m j}\left(\alpha_{i k}+\lambda_{k i}\right)-g_{m k}\left(\alpha_{i j}+\lambda_{j i}\right)+g_{i k}\left(\alpha_{m j}+\lambda_{j m}\right) & -g_{i j}\left(\alpha_{m k}+\lambda_{k m}\right) \\
& +2 g_{m i} \nabla_{[j} \mu_{k]}=0 .
\end{aligned}
$$

From (3.58), it follows that $\alpha_{i k}+\lambda_{k i}=0$ and $\nabla_{[j} \mu_{k]}=0$ simultaneously.

Conversely, using (3.27),

$$
\bar{R}_{k j i m}=W_{k j i m}+Q_{k j i m}-\alpha_{i j} g_{m k}+\alpha_{i k} g_{m j}-\alpha_{m k} g_{i j}+\alpha_{m j} g_{i k},
$$

and substituting (3.29) in (3.27), we get

$$
\begin{aligned}
\bar{R}_{k j i m}= & W_{k j i m}+g_{m j}\left(\alpha_{i k}+\lambda_{k i}\right)-g_{m k}\left(\alpha_{i j}+\lambda_{j i}\right)+g_{i k}\left(\alpha_{m j}+\lambda_{j m}\right) \\
& -g_{i j}\left(\alpha_{m k}+\lambda_{k m}\right)+2 g_{m i} \nabla_{[j} \mu_{k]} .
\end{aligned}
$$

By using the given assumptions of $\alpha_{i k}+\lambda_{k i}=0$ and $\nabla_{[j} \mu_{k]}=0$, we conclude that

$$
\bar{R}_{k j i m}=W_{k j i m}
$$

Next, we give an example of 3-dimensional $W S(g, w, \pi, \mu)$ with a constant curvature in which components of the torsion tensor, complementary, and recurrency covector fields are chosen specially.

Example 3.6 Let us consider the three dimensional metric given as

$$
d s^{2}=\frac{d r^{2}}{1-\kappa r^{2}}+r^{2}\left(d \theta^{2}+\sin ^{2} \theta d \phi^{2}\right), \quad(r>0,0 \leq \theta<\pi, 0 \leq \phi<2 \pi),
$$

where $1-\kappa r^{2}>0$ and $\kappa$ is an arbitrary constant.

The scalar curvature of (3.60) is obtained as $R=6 \kappa$. For $\kappa=1,0,-1$, space is called spherical, planar, and hyperbolic, respectively.

Here, all 1-forms $w, \pi$, and $\mu$ are represented with three components in spherical directions $r, \theta, \phi$, i.e. $w=\left(w_{r}, w_{\theta}, w_{\phi}\right), \pi=\left(\pi_{r}, \pi_{\theta}, \pi_{\phi}\right)$. For this example, we choose the complementary vector $w$ as $w=\left(0, w_{\theta}, 0\right)$, 
ÖZDEMIR and TÜRKOĞLU/Turk J Math

covector fields $\pi$ as $\pi=\left(0, \pi_{\theta}, 0\right)$, and recurrency form $\mu$ as $\mu=\left(0, \mu_{\theta}, 0\right)$, which are defined in equations (2.2), (3.2), and (3.4), respectively. Thus, for the metric (3.60), we find the connection coefficients, the Ricci curvature, and the scalar curvature of $W S(g, w, \pi, \mu)$ as follows:

$$
\begin{array}{ll}
\bar{\Gamma}_{r r}^{r}=\frac{\kappa r}{1-\kappa r^{2}}, & \bar{\Gamma}_{r \theta}^{r}=-w_{\theta}+\mu_{\theta}, \\
\bar{\Gamma}_{\theta r}{ }^{r}=-\left(w_{\theta}+\mu_{\theta}\right), & \bar{\Gamma}_{\theta \theta}^{r}=-r\left(1-\kappa r^{2}\right), \\
\bar{\Gamma}_{\phi \phi}{ }^{r}=-r\left(1-\kappa r^{2}\right) \sin ^{2} \theta, & \bar{\Gamma}_{r r}^{\theta}=\frac{w_{\theta}-\mu_{\theta}}{r^{2}\left(1-\kappa r^{2}\right)}, \\
\bar{\Gamma}_{\phi r}{ }^{\phi}=\bar{\Gamma}_{\theta r}{ }^{\theta}=\bar{\Gamma}_{r \phi}^{\phi}=\bar{\Gamma}_{r \theta}^{\theta}=\frac{1}{r}, & \bar{\Gamma}_{\phi \theta}^{\phi}=\cot \theta-w_{\theta}+\mu_{\theta}, \\
\bar{\Gamma}_{\theta \theta}{ }^{\theta}=-\left(w_{\theta}+\mu_{\theta}\right)=-\pi_{\theta}, & \bar{\Gamma}_{\phi \phi}^{\theta}=-\sin \theta\left(\cos \theta+\left(\mu_{\theta}-w_{\theta}\right) \sin \theta\right), \\
\bar{\Gamma}_{\theta \phi}{ }^{\phi}=\cot \theta-\pi_{\theta} . &
\end{array}
$$

Here, if we choose $w_{\theta}$ and $\mu_{\theta}$ to be functions of radial coordinate $r$, and also $\pi_{\theta}$ to satisfy the relation $\pi_{\theta}=w_{\theta}(r)+\mu_{\theta}(r)$, then from (3.37), we can compute components of the torsion tensor for (3.62).

$$
T_{r \theta}^{r}=T_{\phi \theta}^{\phi}=2 w_{\theta}, \quad T_{\theta r}^{r}=T_{\theta \phi}{ }^{\phi}=-2 w_{\theta},
$$

and the components of the Ricci tensor of $W S(g, w, \pi, \mu)$ are

$$
\begin{aligned}
& \bar{R}_{r r}=\frac{2 \kappa r^{2}+\left(w_{\theta}-\mu_{\theta}\right)\left(\cot \theta-w_{\theta}+\mu_{\theta}\right)}{r^{2}\left(1-\kappa r^{2}\right)}, \\
& \bar{R}_{r \theta}=\frac{2 r w_{\theta}^{\prime}-w_{\theta}+\mu_{\theta}}{r}, \\
& \bar{R}_{\theta r}=-\frac{r\left(w_{\theta}^{\prime}+\mu_{\theta}^{\prime}\right)+w_{\theta}-\mu_{\theta}}{r}, \\
& \bar{R}_{\theta \theta}=2 \kappa r^{2}+\cot \theta\left(w_{\theta}-\mu_{\theta}\right), \\
& \bar{R}_{\phi \phi}=\sin \theta\left(2 \kappa r^{2} \sin \theta+\left(w_{\theta}-\mu_{\theta}\right)\left[2 \cos \theta+\left(\mu_{\theta}-w_{\theta}\right) \sin \theta\right]\right),
\end{aligned}
$$

where prime (') denotes the derivative with respect to $r$, and the scalar curvature of $W S(g, w, \pi, \mu)$ is obtained as

$$
\bar{R}=\frac{1}{r^{2}}\left(6 \kappa r^{2}+2\left(w_{\theta}-\mu_{\theta}\right)\left(2 \cot \theta-w_{\theta}+\mu_{\theta}\right)\right)
$$

Particularly, in (3.64), by taking

$$
w_{\theta}=\lambda_{\theta}=\mu_{\theta}=c_{1} \sqrt{1-\kappa r^{2}}+c_{2}\left[-1+\operatorname{artanh}\left(\frac{1}{\sqrt{1-\kappa r^{2}}}\right)\right], \quad 1-\kappa r^{2}>0
$$

where $c_{1}, c_{2}$ are any real constant, we obtain that the scalar curvature of $W S(g, w, \pi, \mu)$ is $\bar{R}=6 \kappa$. Thus, the scalar curvature of $W S(g, w, \pi, \mu)$ becomes the same as the scalar curvature of Riemannian space.

In the following section, sectional curvature is examined for $W S(g, w, \pi, \mu)$ in the sense of previous studies (see [9], p. 265, and [12]). 
ÖZDEMİR and TÜRKOĞLU/Turk J Math

\subsection{Sectional curvatures on Weyl manifolds with semisymmetric recurrent-metric connection} $W S(g, w, \pi, \mu)$

Let $X, Y \in T_{p}(W S)$ at a point $P \in W S(g, w, \pi, \mu)$. Let $\Pi$ be the 2 -plane spanned by $X, Y$. Then the sectional curvature $K$ of $W S(g, w, \pi, \mu)$ at $P$ with respect to plane $\Pi$ is defined by [12]:

$$
\begin{aligned}
K(\Pi) & =K(X, Y) \\
& =\frac{\bar{R}(X, Y, X, Y)}{g(X, X) g(Y, Y)-g(X, Y)^{2}} .
\end{aligned}
$$

In local coordinates the equation (3.66) is

$$
K(\Pi)=\frac{\bar{R}_{i j k l} X^{i} Y^{j} X^{k} Y^{l}}{\left(g_{i k} g_{j l}-g_{i l} g_{j k}\right) X^{i} Y^{j} X^{k} Y^{l}} .
$$

If the sectional curvature $K$ of $W S(g, w, \pi, \mu)$ at a point $P$ is the same for all 2-planes in $T_{p}(W S)$, then we say that $W S(g, w, \pi, \mu)$ is isotropic at $P$. If $W S(g, w, \pi, \mu)$ is isotropic at every point $P$ on $W S(g, w, \pi, \mu)$, then $W S(g, w, \pi, \mu)$ is called isotropic (see [9], p. 265, and [12]).

On the other hand, if the sectional curvature $K$ of $W S(g, w, \pi, \mu)$ has the same value at every point and for every section at that point, then we say that $W S(g, w, \pi, \mu)$ has constant curvature.

It is obvious that if $W S(g, w, \pi, \mu)$ has constant curvature, then $W S(g, w, \pi, \mu)$ is isotropic.

We recall that a Weyl manifold $W S(g, w, \pi, \mu)$ is said to be an Einstein manifold with respect to the semisymmetric recurrent-metric connection $E W S(g, w, \pi, \mu)$ if the symmetric part of the Ricci tensor is proportional to the metric; that is,

$$
\bar{R}_{(j i)}=\theta g_{i j}
$$

for a scalar function $\theta$ defined on $W S(g, w, \pi, \mu)$ (see [14], Eq. 41).

It is shown that every 2-dimensional Weyl manifold is an Einstein manifold [12].

We now quote the following lemma (see [9], p. 265), which will be needed in the proof of the following theorem.

Lemma 3.7 Suppose that $S$ is any 4-covariant tensor, and that $X$ and $Y$ are two arbitrary linearly independent vectors. If for all $X$ and $Y$

$$
S_{i j k l} X^{i} Y^{j} X^{k} Y^{l}=0
$$

then we have

$$
S_{i j k l}+S_{k l i j}+S_{i l k j}+S_{k j i l}=0 .
$$

A sufficient condition for a Weyl manifold to be locally conformal to an Einstein manifold by using sectional curvature was given in [12]. By means of the notation used in [12], we state and prove the following theorem for $W S(g, w, \pi, \mu)$.

Theorem 3.8 Any isotropic Weyl manifold with the semisymmetric recurrent-metric connection can be locally conformal to an Einstein manifold with the semisymmetric recurrent-metric connection, $E W S(g, w, \pi, \mu)$. 
Proof Assume that $W S(g, w, \pi, \mu)$ is an isotropic manifold. In Lemma 3.7, by taking

$$
S_{i j k l}=\bar{R}_{i j k l}-K(x)\left(g_{i k} g_{j l}-g_{i l} g_{j k}\right)
$$

and using (3.70), we get

$$
\bar{R}_{i j k l}+\bar{R}_{k l i j}+\bar{R}_{i l k j}+\bar{R}_{k j i l}=4 K g_{i k} g_{j l}-2 K\left(g_{l k} g_{i j}+g_{l i} g_{k j}\right) .
$$

Transvecting (3.72) by $g^{l h}$,

$$
\bar{R}_{i j k}{ }^{h}+\bar{R}_{k j i}{ }^{h}+\left(\bar{R}_{k j i l}+\bar{R}_{k j i l}\right) g^{l h}=\left[4 K g_{i k} g_{j l}-2 K\left(g_{l k} g_{i j}+g_{l i} g_{k j}\right)\right] g^{l h}
$$

and using symmetry properties (3.45) and (3.46) and the first Bianchi identity for $E W S(g, w, \pi, \mu)$,

$$
\bar{R}_{k j i}{ }^{l}+\bar{R}_{j i k}{ }^{l}+\bar{R}_{i k j}{ }^{l}=2\left(\delta_{j}^{l} \nabla_{[k} \pi_{i]}+\delta_{i}^{l} \nabla_{[j} \pi_{k]}+\delta_{k}^{l} \nabla_{[i} \pi_{j]}\right)
$$

we find that

$$
\begin{aligned}
\bar{R}_{i j k}{ }^{h}+\bar{R}_{k j i}^{h}+\bar{R}_{i k j}^{h}+2 \bar{R}_{i l k j} g^{l h} & =2 K\left(2 g_{i k} \delta_{j}^{h}-g_{k j} \delta_{i}^{h}-g_{i j} \delta_{k}^{h}\right) \\
& -g^{l h} A_{l k j i}+4 \delta_{j}^{h}\left(\nabla_{[k} w_{i]}+\nabla_{[k} \mu_{i]}\right),
\end{aligned}
$$

where

$$
A_{l k j i}=2\left(g_{i j} \nabla_{[l} \pi_{k]}+g_{k j} \nabla_{[i} \pi_{l]}+g_{l j} \nabla_{[k} \pi_{i]}\right) .
$$

Contracting (3.75) with $h$ and $i$ and using equation (3.74),

$$
\begin{aligned}
\bar{R}_{j k}+\bar{R}_{k j i}^{i}+\bar{R}_{k j}+2 \bar{R}_{i l k j} g^{i l} & =2 K\left(2 g_{i k} \delta_{j}^{i}-g_{k j} \delta_{i}^{i}-g_{i j} \delta_{k}^{i}\right)-g^{l i} A_{l k j i} \\
& +4 \delta_{j}^{i}\left(\nabla_{[k} w_{i]}+\nabla_{[k} \mu_{i]}\right) .
\end{aligned}
$$

For (3.77), by using (3.27), (3.28), and (2.15), let us calculate $\bar{R}_{k j i}^{i}$ and $g^{l i} A_{l k j i}$ :

$$
\bar{R}_{k j i}^{i}=Q_{k j i}^{i}+W_{k j i}^{i}
$$

where

$$
\begin{aligned}
Q_{k j i}^{i} & =\delta_{j}^{i} \lambda_{k i}-\delta_{k}^{i} \lambda_{j i}+\lambda_{j l} g^{l i} g_{k i}-\lambda_{k m} g^{m i} g_{j i}+\delta_{i}{ }^{i}\left(\partial_{j} \mu_{k}-\partial_{k} \mu_{j}\right) \\
& =2 n \nabla_{[j} \mu_{k]}
\end{aligned}
$$

Thus,

$$
\begin{gathered}
Q_{k j i}^{i}=2 n \nabla_{[j} \mu_{k]}, \\
W_{k j i}^{i}=n\left(w_{j k}-w_{k j}\right)=2 n \nabla_{[j} w_{k]},
\end{gathered}
$$


and from (3.76),

$$
A_{l k j i} g^{l i}=2 g^{l i} g_{k j} \nabla_{[i} \pi_{l]}
$$

Hence, we reach

$$
\begin{aligned}
\bar{R}_{j k}+\bar{R}_{k j}+2 n\left(\nabla_{[j} \mu_{k]}+\nabla_{[j} w_{k]}\right) & =2 K\left(2 g_{i k} \delta_{i}^{j}-n g_{k j}-g_{k j}\right) \\
& +4\left(\nabla_{[k} w_{j]}+\nabla_{[k} \mu_{j]}\right)-2 g^{l i} g_{k j} \nabla_{[i} \pi_{l]} .
\end{aligned}
$$

From (3.82), we observe that the symmetric part of the Ricci tensor is

$$
\bar{R}_{(j k)}=(1-n) K g_{k j}-(n+2)\left(\nabla_{[j} w_{k]}+\nabla_{[j} \mu_{k]}\right) .
$$

Since $\bar{R}_{(j k)}$ is symmetric, the second term of (3.83) must satisfy the following relation:

$$
\nabla_{[j} w_{k]}+\nabla_{[j} \mu_{k]}=0
$$

Thus, the symmetric Ricci tensor of $\operatorname{EWS}(g, w, \pi, \mu)$ is

$$
\bar{R}_{(j k)}=(1-n) K g_{k j},
$$

and equation (3.84) implies that $w_{k}$ and $\mu_{k}$ are gradient. This completes the proof.

\section{Acknowledgment}

The authors would like to thank the referees for their valuable comments and suggestions, which helped to improve the paper.

\section{References}

[1] Agashe NS, Chafle MR. A semi-symmetric non-metric connection on a Riemannian manifold. Indian J Pure Appl Math 1992; 23: 399-409.

[2] Eisenhart LP. Non-Riemannian Geometry. Providence, RI, USA: American Mathematical Society, 1927.

[3] Folland GB. Weyl manifolds. J Differential Geometry 1970; 4: 145-153.

[4] Friedmann A, Schouten AJ. Über die Geometrie der Halbsymmetrischen. Übertragungen Mathematische Zeitschrift 1994; 21: 211-233 (in German).

[5] Hayden HA. Subspaces of a space with torsion. Proc Lond Math Soc 1932; 34: 27-50.

[6] Hlavaty V. Theorie D'immersion d'une $W_{m}$ dans $W_{n}$. Ann Polon Math 1949; 21: 196-206 (in French).

[7] Imai T, Yano K. On semi-symmetric metric F-connection. Tensor (NS) 1975; 29: 134-138.

[8] Liang YX. On semi-symmetric recurrent-metric connection. Tensor (NS) 1994; 55: 107-112.

[9] Lovelock D, Rund H. Tensors, Differential Forms and Variational Principles. New York, NY, USA: Dover, 1989.

[10] Norden A. Affinely Connected Spaces. Moscow, USSR: GRMFL, 1976.

[11] Norden A. The theory of surfaces of a biaffine space. Seriya Fiziko-Matematicheskie Nauki 1952; 114: 13-38.

[12] Özdeğer A. On sectional curvatures of a Weyl manifold. Proc Japan Acad 2006; 82: 123-125. 
[13] Özdemir F. A note on semi-symmetric spaces with metric F-connection. BSG Proceedings 2012; 19: $100-109$.

[14] Özdemir F, Türkoğlu MD. On the existence of Einstein Weyl manifold with a special metric connection. In: ICRAPAM 2017; Aydın, Turkey; 2017. p. 159.

[15] Özdemir F, Uysal A. On D-recurrent spaces with semi-symmetric recurrent-metric connection. International Mathematical Forum 2009; 4: 757-765.

[16] Rosen R. Weyl's geometry and physics. Foundations Of Physics 1982; 12: 213-248.

[17] Ünal F, Uysal A. Weyl manifolds with semi-symmetric connection. Mathematical Computational Applications 2005; 10: 351-358.

[18] Yano K. On semi-symmetric metric connection. Rev Roumaine Math Pures Appl 1970; 15: 1579-1586. 